ИЗВЕСТИЯ АКАДЕМИИ НАУК ЭСТОНСКОП ССР. ФИЗИКА * МАТЕМАТИКА PROCEEDINGS OF THE ACADEMY OF SCIENCES OF THE ESTONIAN SSR. PHYSICS * MATHEMATICS

$1988,37,1$

\title{
АССОЦИАТИВНАЯ ЗАПИСЬ ПРОСТРАНСТВЕННО-ВРЕМЕННЫХ ГОЛОГРАММ В ВЫСОКОСЕЛЕКТИВНЫХ ФОТОХРОМНЫХ СРЕДАХ
}

A. REBANE. ASSOTSIATIIVNE AJALIS-RUUMILINE HOLOGRAAFIA KORGSELEKTIIVSETES FOTOKROOMSETES KESKKONDADES

A. REBANE. ASSOCIATIVE RECALL OF TIME-AND-SPACE DOMAIN HOLOGRAMS IN SPECTRALLY SELECTIVE PHOTOACTIVE MEDIUM

\section{(Представил К. К. Ребане)}

Пространственно-временная голография на основе фотовыжигания спектральных провалов является эффективным методом записи и восстановления пространственно-временных характеристик импульсных световых сигналов субнаносекундной длительности $\left[{ }^{1-5}\right]$. Эффект фотовыжигания обеспечивает запись интенсивностей квазимонохроматических компонентов объектного светового сигнала, а использование дополнительного опорного светового сигнала позволяет фиксировать и относительные фазы квазимонохроматических компонентов объектного поля.

В $\left[{ }^{4,5}\right]$ была изложена теория пространственно-временной голографии импульсных световых полей в высокоселективных фотохромных средах в предположении, что роль опорного сигнала выполняется специальным опорным импульсом с плоским волновым фронтом и с малой по сравнению с объектным импульсом длительностью. Рассматривалась схема записи голограмм, где опорный импульс подавался на регистрирующую фотохромную пластинку под определенным углом и с определенной временной задержкой относительно объектного импульса. Считывание голограммы осуществлялось путем подачи повторного короткого опорного импульса с плоским волновым фронтом.

В данном сообщении проведенный в [4,5] анализ обобщается на случай т. н. ассоциативной схемы записи голограмм, где в качестве опорных сигналов служат выделенные отрезки (фрагменты) записываемого объектного светового поля.

Запись ассоциативной пространственно-временной голограммы производится с помощью взаимодействия в спектрально-высокоселективной фотохромной среде разных участков объектного светового поля, а считывание осуществляется путем повторного предъявления какоголибо пространственно-временного участка записанного сигнала по аналогии с ассоциативным считыванием обычных пространственных голограмм [ $\left.{ }^{6}\right]$.

Рассмотрим оптическую схему, где высокоселективная фотохромная пластинка расположена в задней фокальной плоскости $Z$ тонкой сферической фокусирующей линзы с фокусным расстоянием $F$ (см. рисунок). Предположим, что на выходную фокальную плоскость $X$ линзы падает объектный световой импульс длительностью $\Delta t_{\mathrm{S}}$ и шириной 


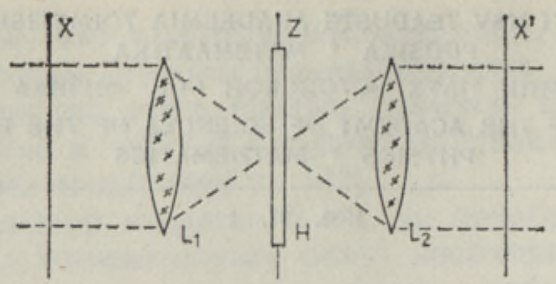

Оптическая схема записи ассоциативной пространственно-временной голограммы. $H$ - голограмма, $L_{1}, L_{2}-$ Фурье-преобразующие линзы, $X$ - входная фокальная плоскость, $X^{\prime}$ - выходная фокальная плоскость, $Z$ - плоскость пространственного Фурье-преобразования.

спектра $\Delta \omega_{\mathrm{S}}$. Запишем амплитуду объектного импульса в виде совокупности $N$ пространственно-временных фрагментов

$$
S(\vec{x}, t)=\sum_{k=1}^{N} s_{k}\left(\vec{x}-\vec{x}_{k}, t-t_{k}\right) e^{i \omega_{0} t}, \quad\left(t_{k}>t_{k-1}\right)
$$

где $s_{k}(\vec{x}, t)$ - комплексная амплитуда $k$-го фрагмента, медленно меняющаяся по сравнению с несущей оптической частотой $\omega_{0}, \vec{x}-$ координата в плоскости $X, \vec{x}_{k}$ - точка отсчета координаты $k$-го фрагмента и $t_{k}$ - временная задержка $k$-го фрагмента относительно переднего фронта объектного импульса.

Предположим, что временная задержка между любыми из фрагментов объектного импульса больше времени корреляции объектного светового поля * $t_{c} \cong \Delta \omega_{\mathrm{s}}{ }^{-1}$ и что длительность объектного импульса в сумме много меньше характерного времени фазовой релаксации $T_{2}$ фотохромной среды.

Следуя общим принципам записи и считывания голограмм в высокоселективных фотохромных средах [4,5], предположим, что ширина спектра объектного импульса не превышает неоднородную ширину полосы поглощения фотохромной среды и что амплитуда падающего на регистрирующую среду светового поля достаточно мала. Тогда нелинейными эффектами (типа насыщения поглощения) можно пренебречь. Далее предположим, что оптическая толщина регистрирующей пластинки-голограммы велика и запись голограммы не изменяет значительно ее спектральное пропускание.

B [ $\left.{ }^{4,5}\right]$ было показано, что при выполнении указанных выше условий, запись голограммы на фотохромной пластинке можно описать при помощи комплексного пространственно-спектрального коэффициента пропускания, величина которого линейно зависит от интенсивности записывающего голограмму светового поля.

В данной схеме записи, где регистрирующая пластинка-голограмма расположена относительно входного сигнала в плоскости пространственного Фурье-преобразования, записывающее непосредственно голограмму световое поле определяется как пространственный Фурьеобраз амплитуды (1) и коэффициент пропускания голограммы будет иметь вид

* Это условие принято для упрощения математических выкладок за счет исключения из рассмотрения пространственной интерференции между разными фрагментами и не является, вообще говоря, принципиальным, 


$$
\begin{gathered}
K(\vec{z}, \omega)=\beta\left[1+\frac{x}{2}(1+i \hat{H})\{I(\vec{z}, \omega)\}\right]=\beta\left[1+\frac{x}{2} \sum_{k=1}^{N}\left|\sigma_{k}\left(\omega-\omega_{0}\right)\right|^{2}+\right. \\
+x \sum_{m=2}^{N} \sum_{k=1}^{m-1} \sigma_{k}^{*}\left(\omega-\omega_{0}\right) \sigma_{m}\left(\omega-\omega_{0}\right) \exp \left\{-i\left[\left(t_{m}-t_{k}\right)\left(\omega-\omega_{0}\right)-\right.\right. \\
\left.\left.-\frac{\omega_{0} z}{c F}\left(\vec{x}_{m}-\vec{x}_{k}\right)\right]\right\},
\end{gathered}
$$

где $I \vec{z}, \omega)$ - спектральная интенсивность светового сигнала, $\hat{H}$ преобразование Гильберта, $c$ - скорость света, $\beta, x-$ определенные в $\left[{ }^{4}\right]$ характеризующие фотохромную среду постоянные и $\sigma_{k}-$ пространственно-временной Фурье-образ амплитуды $k$-го фрагмента:

$$
\sigma_{k}(\vec{z}, \omega)=\iint s_{k}(\vec{x}, t) \exp \left[-i\left(\omega t-\frac{\omega_{0} \vec{z} \vec{x}}{c F}\right)\right] d \vec{x} d t
$$

Если при записи голограммы характерные интервалы пространственных частот разных фрагментов в значительной степени перекрываются, то интерференционный член в (2) будет содержать полную информацию как о пространственной, так и о временной форме объектного импульса.

Для считывания голограммы подадим на вход оптической системы световой импульс, представляющий, для определенности, совокупность $M$ первых фрагментов объектного импульса.

Амплитуда восстановленного с голограммы светового сигнала непосредственно за голограммой будет пропорциональной произведению амплитуды действующего на голограмму считывающего светового поля на комплексный коэффициент пропускания голограммы (2). Для сопоставления восстановленного с голограммы сигнала с исходным объектным импульсом (1) удобно предположить, что за голограммой симметрично первой линзе находится вторая линза (см. рисунок), осуществляющая в своей задней фокальной плоскости $X^{\prime}$ обратное пространственное Фурье-преобразование.

Опуская в (2) не содержащие существенной информации слагаемые и множители, запишем амплитуду восстановленного с голограммы сигнала в плоскости $X^{\prime}$ в виде:

$$
\begin{gathered}
S\left(\vec{x}^{\prime}, t\right)=\sum_{k^{\prime}=1}^{M} \sum_{m=2}^{N} \sum_{k=1}^{m-1} s_{m}\left(\vec{x}-\vec{x}_{m}+\vec{x}_{k}-\vec{x}_{k^{\prime}}, t-t_{m}+t_{k}-t_{k^{\prime}}\right) \otimes \\
\otimes\left[s_{k^{\prime}}\left(\vec{x}^{\prime \prime}, t\right) * s_{k}^{*}\left(\vec{x}^{\prime \prime}+\vec{x}, t^{\prime}+t\right)\right] e^{i \omega_{0} t},
\end{gathered}
$$

где $\otimes$ обозначает интегральную операцию свертки и $*$ - операцию корреляции по пространственной и временной координатам.

Если пространственно-временная структура медленно меняющихся амплитуд фрагментов объектного импульса (1) имеет нерегулярный характер**, то заключенную в квадратные скобки корреляционную функцию можно приблизительно заменить на $\delta$-функцию по пространственной и временной координатам, в результате чего (4) можно будет переписать в виде

** Примером может служить лазерный импульс, рассеянный в диффузной среде, а также импульс, прошедший через отрезок многомодового световода. 


$$
\begin{gathered}
S\left(\overrightarrow{x^{\prime}}, t\right)=\sum_{k=2}^{M} a_{k-1} s_{k}\left(\overrightarrow{x^{\prime}}-\vec{x}_{k}, t-t_{k}\right) e^{i \omega_{0} t}+ \\
+\sum_{k=M+1}^{N} a_{M} s_{k}\left(\vec{x}^{\prime}-\vec{x}_{k}, t-t_{k}\right) e^{i \omega_{0} t}
\end{gathered}
$$

где $a_{k}-$ постоянная, пропорциональная сумме интенсивностей $k$ первых фрагментов.

Сравнивая (5) с первоначальной амплитудой объектного импульса (1), видим, что второе слагаемое в (5) соответствует восстановлению отсутствующих в считывающем импульсе фрагментов объектного сигнала, а первое слагаемое описывает повторное воспроизведение тех фрагментов, которые сами уже входят в состав считывающего импульса. При этом амплитуда восстановленного сигнала возрастает с увеличением числа считывающих фрагментов, что, в свою очередь, можно интерпретировать как свойство ассоциативной памяти восстанавливать детали записанного образа в зависимости от количества предъявляемой для считывания и сравнивания информации.

Отметим, что как следствие причинности пространственно-временных голограмм, гарантируемой в (2) обрезающим действием опера-

тора $(1+i \hat{H})\left[{ }^{4,5}\right]$, степень восстановления объектного сигнала зависит от временной последовательности считывающих и восстанавливаемых фрагментов. Для полного восстановления требуется считывающий фрагмент, который опережал бы остальную часть сигнала во времени.

В заключение отметим, что основные выводы данной работы согласуются с опубликованными в $\left[{ }^{7-9}\right]$ первыми экспериментальными результатами по ассоциативной пространственно-временной голографической записи пикосекундных световых сигналов.

Укажем также, что возможность ассоциативной записи и восстановления меняющихся во времени сигналов отмечалась уже и ранее $\left[{ }^{10}\right]$, причем была указана существующая аналогия между ассоциативной голограммой и некоторыми свойствами человеческой памяти.

Автор выражает искреннюю благодарность К. К. Ребане и П. Саари за полезное обсуждение данной работы.

\section{ЛИТ Е РА Т У Р А}

1. Ребане А. К., Каарли Р. К., Саари П. М. Опт. и спектр., 55, № 3, 405-407 (1983).

2. Ребане А. К., Каарли Р. К., Саари П. М. Пнсьма в ЖЭТФ, 38, вып. 7, 320-323 (1983).

3. Saari, P., Kaarli, R., Rebane, A. J. Opt. Soc. Amer. B, 3, № 4, 527-534 (1986).

4. Саари П. М., Ребане А. К. Изв. АН ЭССР. Физ. Матем., 33, № 3, 322-332 (1984).

5. Саари П. М., Каарли Р. Н., Ребане А. К. Квант. электрон., 12, № 2, 672-682 (1985).

6. Сороко Л. М. Основы голографии и когерентной оптики. М., «Наука», 1971.

7. Ребане А. К., Каарли Р. К. Изв. АН ЭССР. Физ. Матем., 36, вып. 2, 208-212 (1987).

8. Ребане A. K. Мат. симп. «Новые методы лазерной спектроскопии молекул в низкотемпературных средах». Таллин, 1987.

9. Ребане А. К. Мат. V Междунар. симп. «Сверхбыстрые процессы в спектроскопии». Вильнюс, 1987.

10. Gabor, D. Nature, № 217, 1288 (1968).

Институт физики

Академии наук Әстонской ССР
Поступнла в редакцию 2/IX 1987 